\title{
Palatine Bone
}

National Cancer Institute

\section{Source}

National Cancer Institute. Palatine Bone. NCI Thesaurus. Code C52745.

An irregularly shaped bone positioned at the back part of the nasal cavity between the maxilla and the pterygoid process of the sphenoid. It forms the posterior part of the hard palate and the lateral wall of the nasal fossa and helps to form the floor of the orbit as well as several adjoining parts. 